\title{
Mechanical haemolysis in shock wave lithotripsy (SWL): I. Analysis of cell deformation due to SWL flow-fields
}

\author{
Murtuza Lokhandwalla ${ }^{1}$ and Bradford Sturtevant \\ Graduate Aeronautical Laboratories, California Institute of Technology, Pasadena, CA, USA \\ E-mail: murtuza@galcit.caltech.edu
}

Received 5 September 2000, in final form 13 November 2000

\begin{abstract}
This work analyses the interaction of red blood cells (RBCs) with shock-induced and bubble-induced flows in shock wave lithotripsy (SWL), and calculates, in vitro, the lytic effects of these two flows. A well known experimentally observed fact about RBC membranes is that the lipid bilayer disrupts when subjected to an areal strain $(\Delta A / A)_{c}$ of $3 \%$, and a corresponding, critical, isotropic tension, $T_{c}$, of $10 \mathrm{mN} \mathrm{m}^{-1}\left(1 \mathrm{mN} \mathrm{m}^{-1}=1\right.$ dyne $\left.\mathrm{cm}^{-1}\right)$. RBCs suspended in a fluid medium tend to deform in accordance with the deformation of the surrounding fluid medium. The fluid flow-field is lytically effective if the membrane deformation exceeds the above threshold value.

From kinematic analysis, motion of an elementary fluid particle can always be decomposed into a uniform translation, an extensional flow (e.g. $\left.\vec{u}^{\infty}(x, y, z)=(k(t) x,-k(t) y, 0)\right)$ along three mutually perpendicular axes, and a rigid rotation of these axes. However, only an extensional flow causes deformation of a fluid particle, and consequently deforms the RBC membrane. In SWL, a fluid flow-field, induced by a non-uniform shock wave, as well as radial expansion/implosion of a bubble, has been hypothesized to cause lysis of cells. Both the above flow-fields constitute an unsteady, extensional flow, which exerts inertial as well as viscous forces on the RBC membrane. The transient inertial force (expressed as a tension, or force/length), is given by $T_{\text {iner }} \sim$ $\rho r_{c}^{3} k / \tau$, where $\tau$ is a timescale of the transient flow and $r_{c}$ is a characteristic cell size. When the membrane is deformed due to inertial effects, membrane strain is given by $\triangle A / A \sim k \tau$. The transient viscous force is given by $T_{\mathrm{visc}} \sim$ $\rho(\nu / \tau)^{1 / 2} r_{c}^{2} k$, where $\rho$ and $\nu$ are the fluid density and kinematic viscosity.

For the non-uniform shock, the extensional flow exerts an inertial force, $T_{\text {iner }} \approx 64 \mathrm{mN} \mathrm{m}^{-1}$, for a duration of $3 \mathrm{ns,} \mathrm{sufficient} \mathrm{to} \mathrm{induce} \mathrm{pores} \mathrm{in} \mathrm{the} \mathrm{RBC}$ membrane. For a radial flow-field, induced by bubble expansion/implosion, the inertial forces are of a magnitude $100 \mathrm{mN} \mathrm{m}^{-1}$, which last for a duration of $1 \mu \mathrm{s}$, sufficient to cause rupture. Bubble-induced radial flow is predicted to be
\end{abstract}

\footnotetext{
1 Address for correspondence: 205-45 Caltech, Pasadena, CA 91125, USA. See also:

http://www.galcit.caltech.edu/ murtuza
} 
lytically more effective than shock-induced flow in typical in vitro experimental conditions.

\section{List of symbols}

\begin{tabular}{|c|c|}
\hline$\delta_{\omega}$ & boundary layer thickness \\
\hline$\gamma$ & ratio of specific heats \\
\hline$\omega$ & vorticity \\
\hline$\mu$ & viscosity (Pa s) \\
\hline$v$ & kinematic viscosity $\left(\mathrm{m}^{2} \mathrm{~s}^{-1}\right)$ \\
\hline$\phi$ & velocity potential \\
\hline$\rho$ & density $\left(\mathrm{kg} \mathrm{m}^{-3}\right)$ \\
\hline$\tau_{w}$ & tangential wall stress \\
\hline$U_{\text {cell }}$ & cell centre velocity \\
\hline$c$ & acoustic speed $\left(\mathrm{m} \mathrm{s}^{-1}\right)$ \\
\hline$h_{c}$ & cell width \\
\hline$k$ & rate of flow-field deformation $\left(\mathrm{s}^{-1}\right)$ \\
\hline$p_{\text {shock }}$ & shock pressure \\
\hline$p$ & pressure \\
\hline$p_{0}$ & ambient pressure \\
\hline$p_{\infty}$ & far-field pressure \\
\hline$p_{g}$ & gas pressure inside bubble \\
\hline$r_{p}$ & pore radius \\
\hline$r, z$ & cylindrical coordinates \\
\hline$r_{c}$ & cell radius $(\mu \mathrm{m})$ \\
\hline$t, \tau$ & time $(s)$ \\
\hline$u, U$ & velocity in fixed frame \\
\hline$u_{r_{R}}, u_{z_{R}}$ & velocity components (in moving reference frame) \\
\hline$u_{R}$ & velocity of moving reference frame \\
\hline$A$ & cell surface area \\
\hline$(\Delta A / A)_{c}$ & critical membrane areal strain \\
\hline$(\Delta A / A)_{p}$ & membrane strain due to a pore \\
\hline$E_{s}$ & surface elasticity modulus $\left(\mathrm{mN} \mathrm{m}^{-1}\right)$ \\
\hline$M_{s}$ & shock Mach number \\
\hline$Q(t)$ & source/sink strength \\
\hline$R$ & radial coordinate \\
\hline$R_{b}$ & bubble radius \\
\hline$R_{\text {cell }}$ & radial location of cell centre \\
\hline$T$ & membrane tension $\left(\mathrm{mN} \mathrm{m}^{-1}\right)$ \\
\hline$T_{c}$ & critical membrane tension \\
\hline$T_{\text {elas }}$ & elastic tension \\
\hline$T_{\text {iner }}$ & inertial tension \\
\hline$T_{\text {st }}, T_{\text {unst }}$ & steady/unsteady tension \\
\hline$T_{\text {visc }}$ & viscous tension \\
\hline$U_{b}$ & bubble wall velocity \\
\hline
\end{tabular}




\section{Introduction}

Shock wave lithotripsy (SWL) is a non-invasive clinical treatment for renal and ureteral calculi. In this treatment a focused shock wave propagates through about $5 \mathrm{~cm}$ of tissue material before hitting the stone. Various biological effects due to the interaction of this high-amplitude pressure pulse with tissue have been reported (Coleman and Saunders 1993). Studies on animal models have shown that tissue damage in SWL ranges from damage at the cellular level to full-thickness tearing of macrolevel anatomical structures like arteries and veins (Evan and McAteer 1996, Kaude et al 1985).

In this work we aim to understand the interaction of shock and tissue at a mechanistic level. Cellular-level damage occurring in kidney tissue due to SWL has been modelled by RBC damage. Application of shock waves to isolated RBCs in vitro has been reported to cause disruption of the cell membrane and release of cell contents. The fluid flow-field deforms the cell membrane to the extent that the integrity of the membrane is lost, rendering it permeable to water and macromolecules for a sufficient period of time. Thus, the amount of haemoglobin released by the cells after exposure to shock waves is a measure of haemolysis. Cell lysis experiments in SWL implicate two distinct mechanisms: a direct shock-induced effect (Williams et al 1999, Lokhandwalla et al 2001) and an indirect effect due to acoustically induced bubble activity (Delius 1997, Stonehill et al 1998).

In a companion paper (Lokhandwalla et al 2001) we present a novel experimental set-up in which the lithotripter shock wave is refocused by a parabolic reflector, resulting in a wavefield with an increased amplitude and a smaller focal beam-width. RBCs subjected to this refocused shock wave, under conditions of high ambient pressure, show significantly higher haemolysis than with the original lithotripter shock wave. This suggests that the shock-induced flow-field causes the cell membrane to rupture, even in the absence of cavitation. Similar evidence (destruction of cellular material/uptake of molecules by the cell) is also obtained from experiments other than SWL, involving shock waves which do not have cavitation effects (e.g. Doukas et al 1995, Mulholland et al 1999, Teshima et al 1995). In earlier work (Howard and Sturtevant 1997), tissue damage was attributed to the shearing effect of a non-uniform shock. Though this is generally true, in this work we present a more general analysis of a nonplanar, non-uniform shock (for example a focused shock) interacting with a deformable body.

Cavitation-induced cell lysis has been investigated in several experimental works. Experiments (Delius 1997, Stonehill et al 1998) with an increased ambient pressure, reducing cavitation activity, have shown to cause a reduction in cell injury. Though cell lysis strongly correlates with bubble activity, the actual damage mechanism is not understood. Microjetting, occurring due to an asymmetrically imploding cavity, has been commonly cited as a tissue damage mechanism. Microjetting is a manifestation of the instability of the collapsing cavity, and is enhanced by the presence of a rigid wall in the vicinity of the imploding cavity (Blake and Gibson 1987) or by pressure gradients. Most in vitro experiments are designed such that rigid walls are far from the focal region of the lithotripter. Also, there are no pressure gradients at the focus during the collapse phase of the cavities (typically, cavities collapse about $300 \mu$ s after the shock wave has propagated past the focus-see Lokhandwalla et al (2001) and Cleveland et al (2000a)). Hence microjetting may not be a dominant mechanism. Other mechanisms suggested in the literature, such as microstreaming due to interaction of a sound field with bubbles, bubble translation etc, require a continuous-wave $(\mathrm{CW})$ field. Clinically, shock wave dosage is provided at a typical pulse repetition rate of $1 \mathrm{~Hz}$, and hence the above mechanisms are not applicable to SWL.

In this work we propose that significant cell damage can occur due to a radial flow-field, induced by an expanding/collapsing bubble located outside the cell and at a sufficiently large 


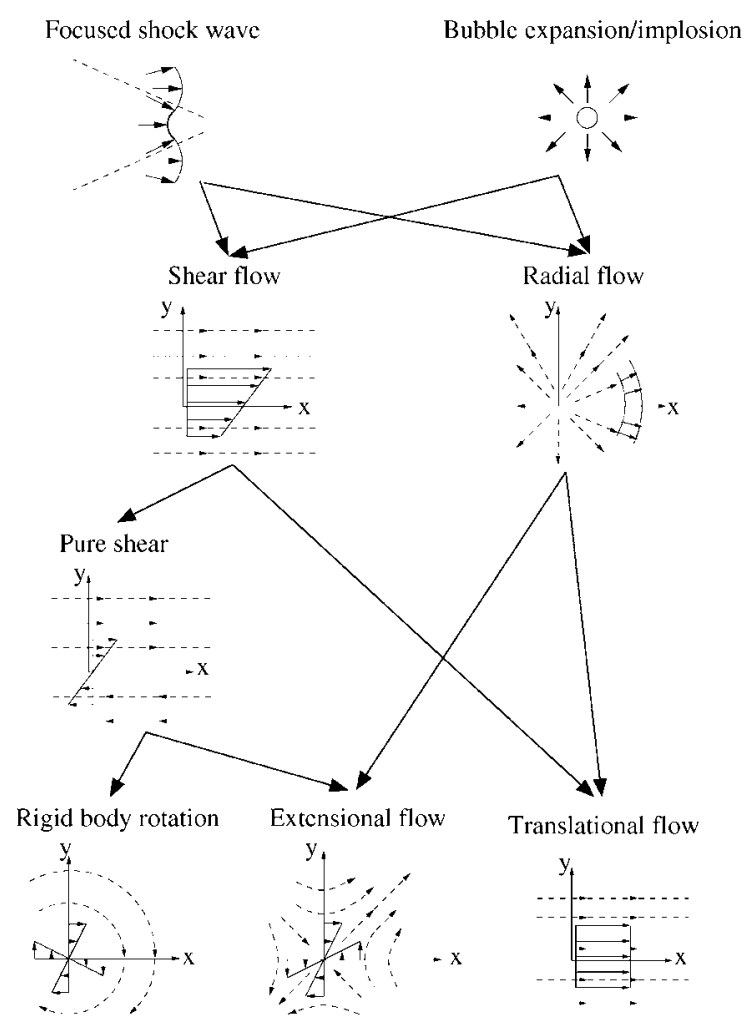

Figure 1. Kinematics of flow induced by focused shock wave and bubble motion. Solid arrows are velocity profiles and dashed arrows are streamlines for each flow field.

distance from it. Cell damage due to radial motion of the extracellular flow-field has already been suggested earlier (Carstensen et al 1993, Miller et al 1996), but a detailed interaction of this extracellular flow-field with a cell is presented here for the first time. The objective of this work is to analyse deformation of a cell subjected to a general flow-field. This general analysis is then applied to the particular case of in vitro exposure of RBCs to SWL shock waves. Membrane stresses and strains due to a focused shock wave and radial bubble motion are evaluated. Though the focus of this work is to evaluate SWL-induced damage, this analysis is sufficiently general, and can be extended to evaluate lytic effects of flow-fields in applications other than SWL.

Most studies of the mechanical properties of RBC membranes continue to support a hypothesis first enunciated by Rand and Burton (1964), that the RBC membrane can withstand a finite strain, beyond which it ruptures. Subsequent work (Evans et al 1976) has confirmed this, and has measured a critical areal strain of $2-3 \%$, beyond which the membrane ruptures. A critical, isotropic (biaxial) tension, $T_{c}$, of $10 \mathrm{mN} \mathrm{m}^{-1}$ is needed to cause this rupture. Hence the question posed is: can SWL flow-fields cause the membrane to deform beyond the threshold strain, and exert the necessary tension? The first step in answering this question involves decomposition of the flow-field into constituent translational, extensional and rotational flow-fields. Since only an extensional flow causes the cells to deform, the forces exerted on the cell membrane by an extensional flow, and the consequent deformation, are estimated. A flow-field is considered to be lytically effective if it can strain the membrane 
beyond the above cited threshold strain, and exert the critical tension required to induce this deformation.

\subsection{Kinematic decomposition of a fluid flow-field}

In order to evaluate membrane tension, induced by a fluid flow-field, it is necessary to decompose a complex flow-field into more elementary forms, as illustrated in figure 1 . The shock wave induces a shear flow due to the velocity gradient along the shock front and a radial flow due to curvature of the wavefront. The shear flow can be decomposed into a uniform translational flow and a 'pure shear' component. The 'pure shear' component further comprises a rigid body rotation and an extensional flow component. Radial motion due to symmetrical bubble expansion/implosion is decomposed into a uniform translational flow and an extensional flow component. Asymmetrical bubble expansion/implosion will cause a jetlike flow, equivalent to a shear flow. Thus the flow-field due to both mechanisms can be decomposed into a translational flow, a rigid body rotation and an extensional flow. This decomposition of flow-field is due to the so called Cauchy-Stokes decomposition theorem: an arbitrary instantaneous state of motion may be resolved at each point into a uniform translation, a dilatation along three mutually perpendicular axes, and a rigid rotation of these axes (Truesdell 1954, Aris 1989).

Only the extensional flow causes deformation of an elementary fluid particle, the other two components merely tend to displace and rotate the fluid particle without any deformation. The same holds true for cells suspended in the fluid medium, only the extensional flow-field deforms the cells. The underlying theme of this work is that the two apparently distinct mechanisms of shock-induced and bubble-induced haemolysis are actually identical so far as their mechanistic effects are concerned, i.e. both flow-fields constitute a sufficiently strong extensional flow-field, inducing a membrane tension greater than $T_{c}$ thus causing lysis.

Derivations for the above decompositions have been included in the appendix for ready reference. According to the above hypothesis, lateral gradients and curvature in the shock front are responsible for deformation of the cell membrane and concomitant lysis. Thus, if the shock-front is plane and of uniform strength, a uniform translational flow follows the shock, which according to this hypothesis causes no cell lysis.

\subsection{Cell deformation}

The problem is to describe the interaction between this extensional flow and the cell. The forces acting on the cell wall are due to inertia of the fluid particles $\left(T_{\text {iner }}\right)$ and viscous stresses ( $\left.T_{\text {visc }}\right)$, causing it to deform. The quantity $T_{\text {iner }}$ is a measure of the fluid inertia, expressed as a tension. $T_{\text {visc }}$ is the membrane tension due to tangential viscous stresses, exerted by the fluid, on the cell wall. Deformation of the membrane due to the extensional flow induces an elastic tension, $T_{\text {elas }}$, which opposes the above forces exerted by the flow-field. At any given instance there exists a balance between $T_{\text {elas }}$ and either one or both of the above-mentioned forces. Based on the relative strengths of these forces, this interaction is classified into two limiting cases (as shown in figure 2), denoted as inertial and viscous modes of deformation:

- Inertial mode $\left(T_{\text {elas }} \ll T_{\text {iner }}\right)$ : The cell membrane is very weak compared with the inertial forces, and hence deformation is described by flow kinematics, as shown in figure 2( $a$ ). The presence of the membrane only perturbs the flow in its vicinity. As long as $T_{\text {elas }} \ll T_{\text {iner }}$ a steady-state deformation of the cell cannot be attained, and the cell deformation is identical to that of the fluid particles in absence of the membrane. As a consequence, there are negligible viscous stresses on the cell membrane, i.e. $T_{\text {visc }} \approx 0$. 


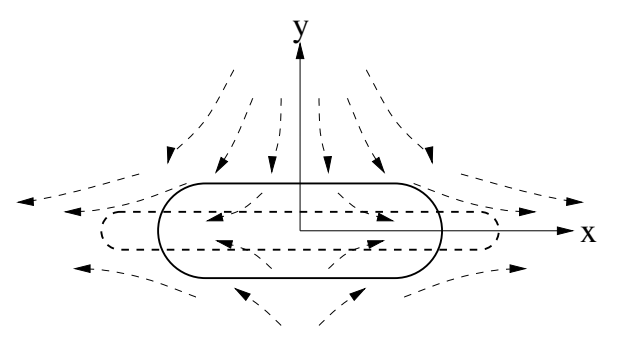

a) $T_{\text {elas }}<<T_{\text {iner }}$

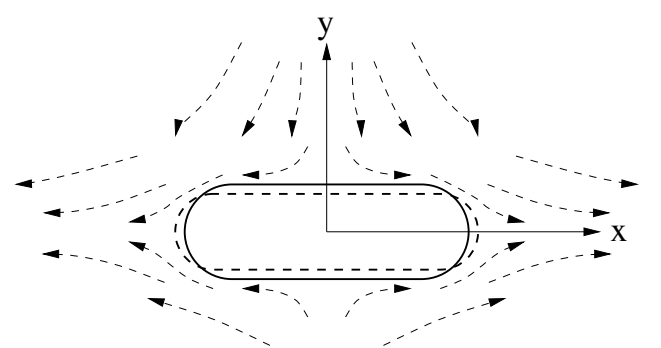

b) $\mathrm{T}_{\text {elas }}>\mathrm{T}_{\text {iner }}$

Figure 2. Cell deformation due to extensional flow. Dashed arrows indicate streamlines. (a) Inertial mode of deformation. (b) Viscous mode of deformation.

- Viscous mode $\left(T_{\text {elas }} \gg T_{\text {iner }}\right)$ : The cell membrane is strong compared with the inertial forces, and hence membrane deformation cannot be described by flow kinematics. The flow-field is still extensional in the far-field, but at the cell wall it has to conform to the no-slip boundary conditions (figure $2(b)$ ). Thus, tangential viscous stresses are exerted on the cell wall, which will be in equilibrium with the membrane tension, $T_{\text {elas }} \sim T_{\text {visc }}$. In this case a steady-state deformation of the cell could exist, provided that the membrane elastic stress does not exceed the threshold value, i.e. $T_{\text {elas }}<T_{c}$.

In the next section the mechanical properties of lipid membranes and the mechanics of membrane poration are briefly reviewed, since they form the basis of our hypothesis about membrane rupture. Calculation of inertial and viscous forces due to an unsteady extensional flow, are presented in sections 3 and 4, respectively. In an unsteady flow-field, the relative magnitudes of the inertial and viscous forces, compared with the elastic membrane tension, can change with time. In such a situation the interaction could change from the inertial mode (figure 2(a)) to the viscous mode (figure $2(b)$ ), or vice versa, as the flow evolves. The transition from inertial mode to viscous mode is discussed in section 5 . The expressions derived in sections 3 and 4 are used in section 6 to calculate the membrane tension due to unsteady extensional flow induced by a focused shock wave and radial bubble expansion. Finally, the limitations of this calculation and our conclusions are presented.

\section{Membrane properties}

For purpose of this analysis, the RBC is structurally equivalent to a fluid-filled capsule with the cell membrane separating the cell contents from its exterior. The shape of the cell is a biconcave disc, with the disc diameter being $8 \mu \mathrm{m}$ and the disc thickness varying from $1 \mu \mathrm{m}$ at the centre to $2.6 \mu \mathrm{m}$ at the periphery. Mechanical properties of the RBC membrane have been investigated in sufficient detail in the past. A simple experiment which shows membrane characteristics is osmotic swelling and subsequent lysis of cells. During osmotic swelling it has been observed (Evans and Fung 1972) that the cell volume increases significantly (75\%), whereas its area remains almost constant (increases by only 7\%). A hypothesis first proposed by Rand (Rand 1964, Rand and Burton 1964), that pores appear in the cell wall when the membrane surface area exceeds a threshold value, has found general acceptance in the literature.

More controlled experiments on a single cell (Evans et al 1976, Needham and Hochmuth 1989) are performed using a micropipette aspirating system in conjunction with an optical processing system (see figure 3). Applying a suction pressure in the micropipette causes an increase in the length of the aspirated part of the cell and a reduction in the cell radius, which 

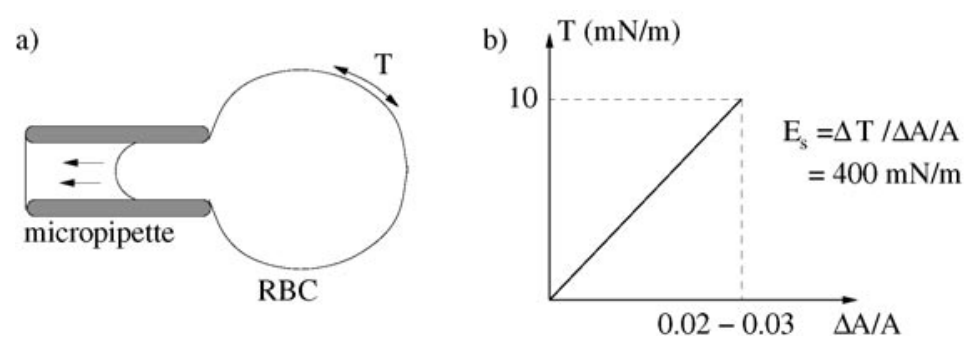

Figure 3. (a) Micropipette aspirator. (b) Linear $T$ versus $\Delta A / A$ relation.

is a consequence of the cell volume remaining approximately constant. These dimensions are measured at a given pressure in the aspirator. The tension, $T$, in the cell wall is calculated from the pressure difference across it, and the radius of the cell. The areal strain $(\Delta A / A)$ is obtained from the length of the cell projection in the pipette (see Evans et al (1976) for details of this calculation). Measurements indicate a linear relation between the membrane tension and the areal strain. The lipid bilayer is treated as a continuum in two dimensions, and is characterized by a surface elasticity modulus $\left(E_{S}=\mathrm{d} T / \mathrm{d} A / A\right)$ and critical values of tension $\left(T_{c}\right)$ and areal strain $(\triangle A / A)_{c}$ for failure. These refined experiments indicate a critical areal strain of $2-3 \%$, a critical tension, $T_{c}$, of $10 \mathrm{mN} \mathrm{m}^{-1}$ and surface elasticity modulus $\sim 400 \mathrm{mN} \mathrm{m}^{-1}$. These membrane properties will be used in subsequent calculations. Besides the micropipette aspirator, another commonly used apparatus for measuring cell membrane properties is a cone-plate or the Couette flow viscometer. In this work, however, results from the experiments with the micropipette aspirator are considered as a basis for comparison. This is done since calculation of membrane tension from these experiments is rather elementary due to the static nature of the forces involved. While studying membrane deformation, it is usual to measure the tension (has units of force/length or stress $\times$ membrane thickness), rather than stress itself (force/area $=$ tension/membrane thickness). Throughout this work, the words tension and stress are used interchangeably, though we always calculate tension (force/length).

Membrane failure at a critical stress/strain is attributed to membrane poration. The formation of pores in the lipid bilayer, under the action of an isotropic tension, has been explained as an electromechanical instability in the membrane. The lipid macromolecule consists of a water-soluble (hydrophilic), polar, head group of molecules, and a non-polar hydrocarbon tail, which is water-insoluble (hydrophobic). In the bilayer configuration, the hydrophilic head groups face the aqueous medium and the hydrophobic tails face inward, or towards each other. This arrangement minimizes the energy of the system and is hence stable. However, when the membrane is subjected to an isotropic tension, the molecules are drawn further apart. The tail of the lipid molecule is exposed to the aqueous medium, forming a hydrophobic pore. Pores smaller than 1-10 nm (Litster 1975) are energetically unfavourable and the membrane heals itself. However, pores larger than the above size grow unstably, leading to membrane rupture.

These pore sizes give an areal strain of $(\Delta A / A)_{p} \sim 10^{-7}-10^{-5}$, which is several orders smaller than the critical strain, measured experimentally $\left(\sim 10^{-2}\right)$. This discrepancy is explained by the fact that the formation of a single or multiple unstably growing pore is a statistical process, i.e. the probability of a pore growing unstably increases with time, membrane tension $T$ (or the corresponding membrane strain $\Delta A / A$ ) and temperature, for a membrane of given composition. For strains $\mathcal{O}(\Delta A / A)_{c}$, the probability that the membrane ruptures is close to unity and is readily observed, whereas for strains $\mathcal{O}(\Delta A / A)_{p}$ this probability is 
negligibly small and hence not observed. Numerical simulations described in literature (e.g. Shillcock and Boal 1996) investigate the effect of the membrane parameters on unstable pore formation.

In electroporation, instead of a mechanically applied tension an electric field is applied across the membrane. This induces induces a compressive stress $\left(\sigma_{e}\right)$ across the membrane thickness, and results in a similar instability (Needham and Hochmuth 1989). Our current understanding about pore formation in cell membranes stems largely from electroporation experiments (see Weaver (1995) for a review of electroporation theory). In these experiments, application of an electric field across the membrane for a short duration, beyond a certain threshold, causes increased transport of molecules across the membrane (Neumann and Rosenheck 1972). Cell death is also observed following the application of an electric field. Cell death can occur either due to rupture of the membrane, i.e. unstable pore formation, or due to significant molecular transport between the intra- and extra-cellular environments, leading to a chemical imbalance from which the cell is unable to recover. Thus, depending upon the parameters of the electric field and the membrane characteristics, the membrane is either transiently permeabilized or irreversibly ruptured. By analogy, deformation of a cell membrane induced by fluid flow-field also results in increased transport across the cell membrane or cell lysis, depending on the flow parameters.

\section{Inertial forces}

As explained in section 1.1, and illustrated in figure $2(a)$, in the inertial mode $\left(T_{\text {elas }} \ll T_{\text {iner }}\right)$, membrane deformation is identical to that of an elementary fluid particle. The velocity potential, $\phi(t)$, for such a flow field, is given by

$$
\phi(t)=k(t)\left(\frac{r^{2}}{2}-z^{2}\right) .
$$

Equation (1) can be obtained from (A.12) in appendix A, by neglecting terms $\mathcal{O}\left(z^{3}, z r^{2}\right)$, reverting to the dimensional form, and denoting $u_{R}(t) / R$ as $k(t)$. The parameter $k(t)$ is a kinematic property of the flow-field, and is a measure of the rate of deformation of the flow. For a shear flow $k(t)=\frac{\partial u_{x}}{\partial y}$. The coordinate system adopted in this analysis is fixed with reference to the cell. For any given cell, $k$ depends only on time, as the cell assumes the motion of the fluid particle. Bernoulli's equation for unsteady, incompressible, potential flow, is

$$
\frac{\partial \phi}{\partial t}+\frac{(\nabla \phi)^{2}}{2}+\frac{p}{\rho}=\text { constant }
$$

In the above equation $p$ and $\rho$ are pressure and density respectively, and gravitational potential is ignored. Substituting (1) in (2), we get

$$
\dot{k}(t)\left(\frac{r^{2}}{2}-z^{2}\right)+\frac{k^{2}(t)}{2}\left(r^{2}+4 z^{2}\right)+\frac{p}{\rho}=\text { constant }
$$

Figure 4 shows the configuration of the problem, the disc-shaped cell deforming due to the unsteady extensional fluid flow-field. The potential, $\phi$, given by (1), is assumed to apply to the interior as well as to the exterior of the cell. This requires that the flow is minimally perturbed by the cell membrane. We also assume that $\rho$ is identical for the fluid inside the cell (specific gravity $=1.1$ ), and the suspending medium. Depending on in vivo/vitro condition, this medium is either plasma ( specific gravity $=1.03$ ), or saline solution (specific gravity $\approx 1$ ). 

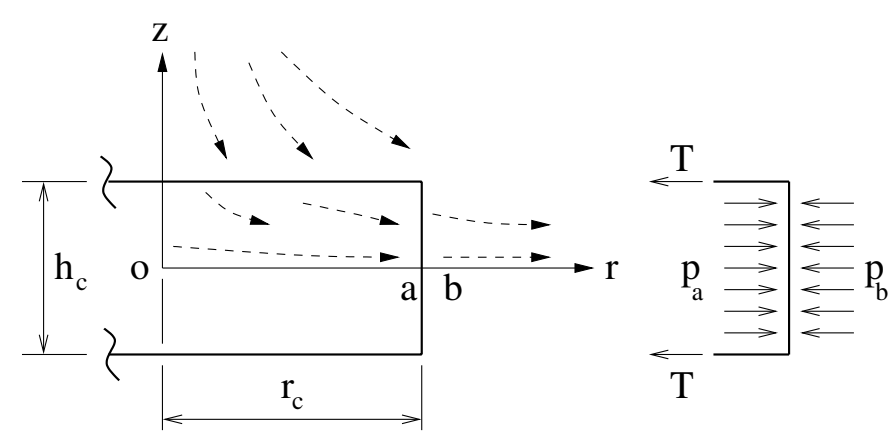

Figure 4. Cell deformation due to inertial force. The cell membrane is indicated by thick lines, and the cell is assumed to be disc-shaped. Dashed arrows indicate the streamline pattern.

Applying (3) to a radial streamline (see figure 4), originating from the origin (o) up to a point just inside the cell (a), we get

$$
\dot{k}(t) \frac{r_{c}^{2}}{2}+\frac{k^{2}(t)}{2} r_{c}^{2}=\frac{p_{o}-p_{a}}{\rho} .
$$

The quantity $k r_{c}$ is a measure of the fluid velocity at the cell wall, in a cell-fixed reference frame. In (4), the left-hand side represents the inertial force of the fluid (temporal and convective acceleration of fluid), balanced by hydrodynamic pressure on the right-hand side. In the absence of the cell membrane the hydrodynamic pressure is continuous at all points in the flow-field, and hence $p_{a}=p_{b}$ (see figure 4) for adjacent points $a$ and $b$. However, in the presence of the membrane, the extensional flow causes the membrane to deform inducing a tension in it, which requires a pressure differential to exist across the membrane in order to balance this elastic tension. This balance is given by

$$
T_{\text {elas }}=\left(p_{a}-p_{b}\right) h_{c} \approx\left(p_{a}-p_{b}\right) r_{c}
$$

where $T_{\text {elas }}$ is the elastic tension in the membrane and $h_{c}$ is the width of the disc, which is approximated by a characteristic cell dimension, $r_{c}$. Using (5), equation (4) can be rewritten as

$$
\frac{r_{c}^{2}}{2}\left(\dot{k}(t)+k^{2}(t)\right)=\frac{p_{o}-p_{b}}{\rho}-\frac{T_{\text {elas }}}{\rho r_{c}} .
$$

Thus from (6), the inertial force of the fluid, expressed as a tension, is given by

$$
T_{\text {iner }} \sim \rho r_{c}^{3}\left(\dot{k}(t)+k^{2}(t)\right) .
$$

The dependence of temporal and spatial acceleration terms on $k(t)$ should be noted here. The temporal term is proportional to $\dot{k} \sim k / \tau$, where $\tau$ is a time scale associated with the unsteady flow, whereas the spatial or convective term is proportional to $k^{2}$. The ratio of temporal to spatial acceleration is $1 / \tau k$ (note that $k$ has a dimension of time ${ }^{-1}$ ). Thus, when the time scale of variation of the unsteady flow-field is smaller than $1 / k,(\tau \ll 1 / k)$, the unsteady inertial force will be predominant, i.e.

$$
T_{\text {iner }} \sim \rho r_{c}^{3} \dot{k}(t) \sim \rho r_{c}^{3} \frac{k}{\tau}
$$

As it will be seen in section 6 , this is indeed the case for a very brief time for shock-induced extensional flow. 


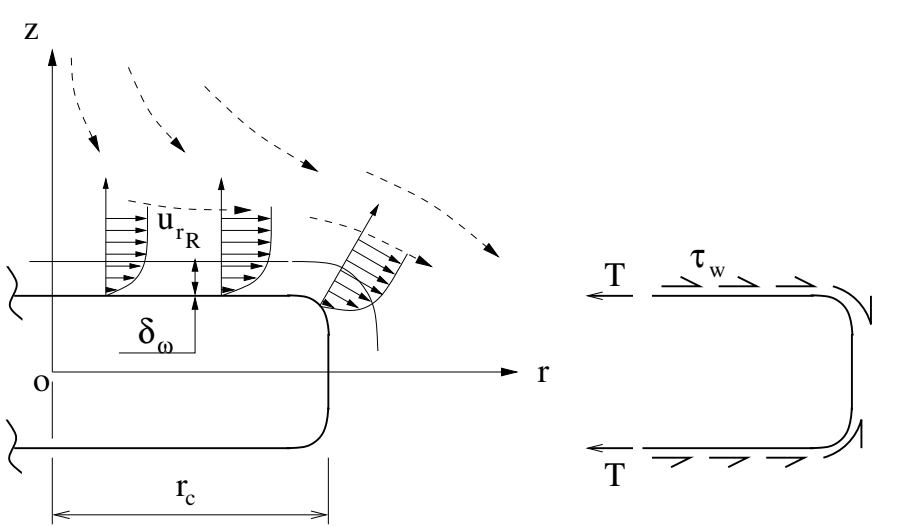

Figure 5. Cell deformation due to viscous force. The cell membrane is indicated by thick lines. Velocity profiles show boundary layer formation. Dashed arrows indicate the streamline pattern for the far-field extensional flow and $u_{r_{R}}$ is the radial velocity tangential to the wall, determined from the far-field flow.

The elastic tension, $T_{\text {elas }}$, actually induced in the membrane is a function of the deformation of the membrane. Since the membrane deforms with the fluid, the radial displacement of a particle on the disc surface (see figure 4) will be given by

$$
\Delta r=\int_{0}^{\tau} u_{r_{R}} \mathrm{~d} t=r \int_{0}^{\tau} k(t) \mathrm{d} t
$$

where $u_{r_{R}}$ is the radial component of velocity (see appendix A for an explanation of notation). Thus, for a disc of radius $r_{c}$, with thickness $h_{c}<r_{c}$, the areal strain is approximately given by

$$
\Delta A / A \approx \Delta r_{c} / r_{c}=\int_{0}^{\tau} k(t) \mathrm{d} t
$$

For an extensional flow which grows linearly from 0 to $k$ in a time $\tau$, the areal strain is estimated as $\sim k \tau$. Consequently, the tension induced in the membrane, $T_{\text {elas }}$, will be $\sim E_{s} k \tau$. These estimates of areal strain and membrane tension hold only for the inertial mode of deformation.

\section{Viscous forces}

In this section we estimate the viscous force $\left(T_{\mathrm{visc}}\right)$ exerted on the cell membrane due to the unsteady extensional flow. As explained in section 1.2, when the inertial forces are weak ( $\left.T_{\text {elas }} \ll T_{\text {iner }}\right)$, the membrane does not deform with the extensional flow-field, rather the extensional flow-field has to obey the no-slip condition at the cell wall. Thus, a transient boundary layer, $\delta_{\omega}$, develops (see figure 5), and a tangential stress, $\tau_{w}$, is exerted on the wall. This tangential stress induces a membrane tension.

Expressions for $\delta_{\omega}$ and $\tau_{w}$ are identical to that for Stokes' first problem, which deals with one-dimensional unsteady flow (see texts in fluid mechanics such as Pozrikidis (1997) or the appendix in Lokhandwalla (2001)). The problem at hand can be assumed to be one-dimensional if the boundary layer width, $\delta_{\omega}$, is smaller than the cell dimension, $r_{c}$. For the unsteady flow the boundary layer width grows with time, $\delta_{\omega}=\sqrt{\pi v t}$, where $v$ is the kinematic viscosity of the suspending medium. Hence the above problem can be treated as a one-dimensional unsteady flow, for $t<r_{c}^{2} /(\pi v) \approx 6 \mu \mathrm{s}$. For $t>6 \mu \mathrm{s}$ the boundary layer becomes larger than the cell size, and the flow-field can be approximated as a quasisteady Stokes' flow. RBCs subjected to quasisteady Stokes' flow have been dealt with in several theoretical and computational works 
(Ramanujan and Pozrikidis (1998), Barthes-Biesel (1980) and Li et al (1988) are a few notable ones). The motivation in these works is to calculate RBC deformation and to explain other features of RBC motion, for instance the tank-treading motion of the cell membrane as it commutes through the blood vessels.

Since the far-field flow is an extensional flow, the velocity component in the radial direction is given by $u_{r_{R}}=k(t) r$ (from (A.9) if terms $\mathcal{O}\left(r^{2}, z^{2}\right)$ are neglected). The definition of $k(t)$ remains same as in the previous section, i.e. $k(t)=u_{R}(t) / R$ for a radial flow and $k(t)=\frac{\partial u_{x}}{\partial y}$ for a shear flow. The tangential wall stress on the cell membrane is given by (see the appendix in Lokhandwalla (2001))

$$
\tau_{w}=\rho \sqrt{\frac{\nu}{\pi}} \int_{0}^{t} \frac{\mathrm{d} U(\tau)}{\mathrm{d} \tau} \frac{1}{\sqrt{(t-\tau)}} \mathrm{d} \tau .
$$

where $U(t)$ is an arbitrary time-dependent velocity of the far-field flow. When the above $u_{r_{R}}$ is substituted in place of $U(t)$ in (11), we obtain

$$
\tau_{w}(r, t)=\rho r \sqrt{\frac{\nu}{\pi}} \int_{0}^{t} \frac{\mathrm{d} k(\tau)}{\mathrm{d} \tau} \frac{1}{\sqrt{(t-\tau)}} \mathrm{d} \tau .
$$

The balance of forces acting on the membrane relates the membrane tension with the above tangential wall stress, and is given by

$$
T_{\text {visc }}(r, t)=\int_{r}^{r_{c}} \tau_{w}(r, t) \mathrm{d} r .
$$

Substituting (12) in (13), we get,

$$
\begin{aligned}
& T_{\text {visc }}(r, t)=\rho \sqrt{\frac{\nu}{\pi}} \int_{r}^{r_{c}} r \mathrm{~d} r \int_{0}^{t} \frac{\mathrm{d} k(\tau)}{\mathrm{d} \tau} \frac{1}{\sqrt{(t-\tau)}} \mathrm{d} \tau \\
& T_{\text {visc }}(0, t)=\frac{\rho r_{c}^{2}}{2} \sqrt{\frac{\nu}{\pi}} \int_{0}^{t} \frac{\mathrm{d} k(\tau)}{\mathrm{d} \tau} \frac{1}{\sqrt{(t-\tau)}} \mathrm{d} \tau .
\end{aligned}
$$

If $k(t)$ grows linearly with time $(=K t)$, then (15) gives

$$
T_{\mathrm{visc}}(0, t)=\rho r_{c}^{2} K \sqrt{\frac{v t}{\pi}}=\rho r_{c}^{2} k(t) \sqrt{\frac{v}{\pi t}} .
$$

Since the elastic tension in the membrane equilibrates with the viscous forces, $T_{\text {elas }} \approx T_{\text {visc }}$, and consequently membrane deformation is proportional to $T_{\text {visc }}$. Thus, critical deformation is reached when $T_{\text {visc }}=T_{c}$.

In contrast with the above estimate of wall tension, for a steady flow wall tension due to viscous force is estimated as

$$
\begin{aligned}
T_{\mathrm{visc}, \mathrm{st}} & \approx \tau_{w} r_{c} \\
& \approx \mu k r_{c} .
\end{aligned}
$$

The steady flow assumes that the flow, characterized by $k(t)$, remains constant for a sufficient time. The ratio $T_{\text {visc,st }} / T_{\text {elas }} \sim \mu k r_{c} / T_{\text {elas }}$ is a counterpart of the capillary number $\left(\mu k r_{c} / \gamma\right)$ associated with bubbles suspended in a shear flow, with the elastic tension replacing the constant surface tension $(\gamma)$ in the denominator.

In table 1, calculations from this and the preceding section have been tabulated. In this table $T_{\text {visc }}$ is calculated assuming a linearly increasing $k(t)$, for a time period $\tau$. The ratio $T_{\text {iner }} / T_{\text {visc }}$ gives the relevant Reynolds number of the flow, for both the cases, i.e. steady and unsteady. This ratio $(R e)$ determines the relative strength of the two forces. The last row tabulates the ratio of unsteady forces to steady forces, $T_{\mathrm{unst}} / T_{\mathrm{st}}$, for the same extensional flow. 
Table 1. Summary table of viscous and inertial tensions, in steady and unsteady flow.

\begin{tabular}{llll}
\hline & $T_{\text {iner }}$ & $T_{\text {visc }}$ & $T_{\text {iner }} / T_{\text {visc }} \sim R e$ \\
\hline Unsteady & $\rho r_{c}^{3} k / \tau$ & $\rho r_{c}^{2} k(\nu / \tau)^{1 / 2}$ & $r_{c} /(\nu \tau)^{1 / 2}$ \\
Steady & $\rho r_{c}^{3} k^{2}$ & $\mu k r_{c}$ & $\left(k r_{c}\right) r_{c} / \nu$ \\
$T_{\text {unst }} / T_{\text {st }}$ & $1 / k \tau$ & $r_{c} /(\nu \tau)^{1 / 2}$ & \\
\hline
\end{tabular}

This ratio can be seen to be proportional to the ratio of a steady time scale to an unsteady time scale (Strouhal number). Thus, if the flow remains steady for a time of the order of the steady time scale, then the steady calculations are valid. Conversely, if the flow varies at a time scale smaller than the steady time scale, then the unsteady calculations presented in this work are valid. For inertial effects, the steady time scale is $1 / k$, whereas for viscous effects the steady time scale is $r_{c}^{2} / \nu$.

\section{Transition from $T_{\text {iner }}$ to $T_{\text {visc }}$}

In the previous two sections, while estimating the inertial and viscous forces the magnitude of the inertial force relative to the elastic tension was assumed to be such that the deformation mode (inertial/viscous) does not change. However, since the flow is unsteady, the relative magnitude of $T_{\text {iner }}$ can change with respect to $T_{\text {elas }}$ with time, causing a transition in the deformation mode as well. In this section we provide a qualitative description of this transition. In the following paragraphs we will assume that $k$ increases linearly with time; however, this description of transition can be generalized to flow-fields which vary arbitrarily with time.

Figure $6(a)$, illustrates a sequence of possible deformation states for a monotonically increasing $k$. The distinct states in this sequence are numbered as: initial state is 0 , lysed states are numbered as 1 or 2 , primed numbers $\left(1^{\prime}, 2^{\prime}, 1^{\prime \prime}\right.$, etc) represent some intermediate state. In figure $6(b)$ the same sequence of deformation is plotted on a $k-\tau$ plane. The line marked as $T_{\text {elas }}=T_{\text {iner }}$ is a solution of the equation $E_{s} k \tau=\rho r_{c}^{3}\left(k / \tau+k^{2}\right)$, and demarcates the two modes of deformation on the $k-\tau$ diagram. The region to the left of the above line implies $T_{\text {elas }}<T_{\text {iner }}$, and hence an inertial mode of deformation. The region to the right of the above line will have $T_{\text {elas }}>T_{\text {iner }}$, and represents the viscous mode of deformation. Lines marked as $T_{\text {iner }}=T_{c}$ and $\Delta A / A=2.5 \%$ are solutions of the equations $\rho r_{c}^{3}\left(k / \tau+k^{2}\right)=0.01$ and $k \tau=0.025$ respectively. The line marked as $T_{\text {visc }}=T_{c}$ is a solution of the equation $\rho r_{c}^{2} k(\nu / \tau)^{1 / 2}+\rho \nu k r_{c}=0.01$. These lines are plotted bold in their respective regimes of validity, and dashed otherwise. For instance, the line $\Delta A / A=k \tau=2.5 \%$ is valid in the inertial deformation mode (shown bold). In the viscous mode $\Delta A / A=k \tau$ is not strictly true and this line (shown dashed) will have a smaller slope (closer to horizontal) than shown. For the lines $T_{\text {iner }}=T_{c}$ and $T_{\text {visc }}=T_{c}$, the rising portions indicate the dominance of the unsteady contribution to the total force, whereas flat portions indicate the contribution of the steady force (independent of $\tau$ ). It should be noted here that only $k$ and $\tau$ are properties of the flow-field; $r_{c}, E_{s}, T_{c}$ and $(\triangle A / A)_{c}$ are attributes of the $\mathrm{RBC}$, and $\rho, v$ are physical properties of the fluid. Physical properties of water are used while plotting the above lines: $\rho=10^{3} \mathrm{~kg} \mathrm{~m}^{-3}$, $\mu=\rho v=10^{-3}$ Pa s.

For all unsteady flow situations, as in the case of SWL, the membrane is initially undeformed, i.e. $T_{\text {elas }}=0$, and hence initial deformation will always be of inertial mode $\left(0-1^{\prime}\right)$. Two possible scenarios exist for subsequent deformation. One possibility is that the inertia of the fluid is strong enough to cause the cell to lyse $\left(1^{\prime}-1\right)$, and viscous forces have no role to play. In the inertial mode, critical deformation is attained for state points above 


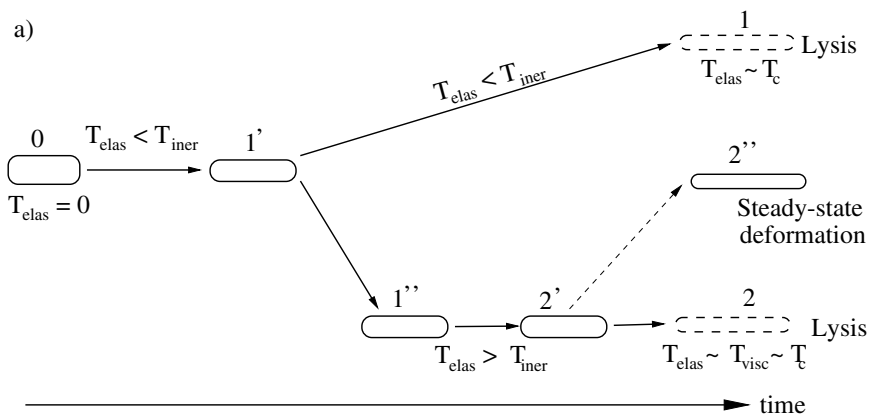

b)

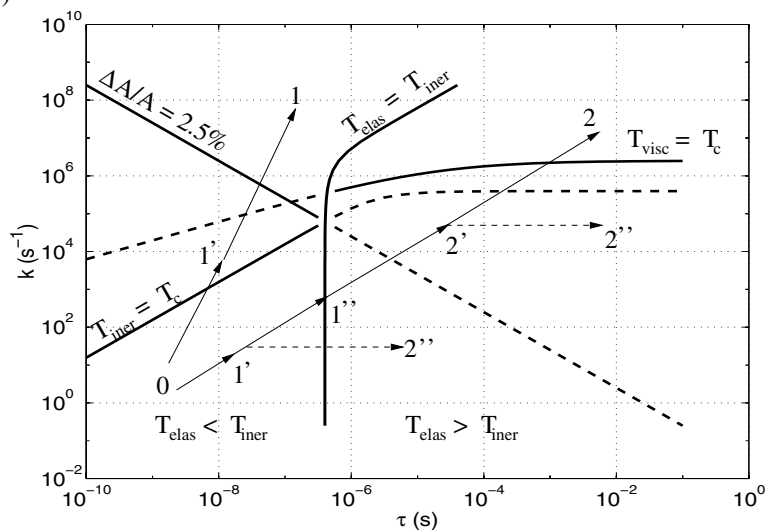

Figure 6. Cell deformation due to unsteady extensional flow. Deformation states of the cell are represented by numbers: $0,1,1^{\prime}$, etc. (a) Sequence of possible deformation states in an unsteady extensional flow. $(b)$ Sequence shown in (a) represented on a $k-\tau$ plane (see text for explanation).

$\Delta A / A=2.5 \%$ (e.g. 1). Alternatively, the membrane is stronger than the inertial forces, then the viscous forces determine the fate of the cell. The transition from inertial to viscous mode will occur gradually, in vicinity of the line $T_{\text {elas }}=T_{\text {iner }}$. However, here we assume that the transition occurs instantaneously, across the above line (e.g. at state point $1^{\prime \prime}$ ). State point $2^{\prime}$ indicates a subcritical deformation, in the viscous mode, whereas state point 2 indicates a critical deformation, sufficient for lysis. In the viscous mode, critical deformation is attained for state points above the line $T_{\mathrm{visc}}=T_{c}$ (e.g. 2). If $k$ is maintained constant (instead of monotonically increasing) after attaining state points $1^{\prime}$ or $2^{\prime}$, then a steady-state deformation of the cell ( $\left.2^{\prime \prime}\right)$ results. In subsequent calculations, the quantities $T_{\text {iner }}$ and $T_{\text {visc }}$ will be calculated without regard to this transition, since such a detailed calculation is beyond the scope of this work.

\section{Application}

In this section we estimate $T_{\text {iner }}$ and $T_{\text {visc }}$ for the shock-induced flow and the bubble-induced flow encountered in SWL, using results from the previous section. As can be seen from equation (7) and (15) derived earlier, the quantity $k(t)$ characterizing the unsteady extensional flow needs to be deduced for each of the flow situations in order to compute the tensions and the deformations. 

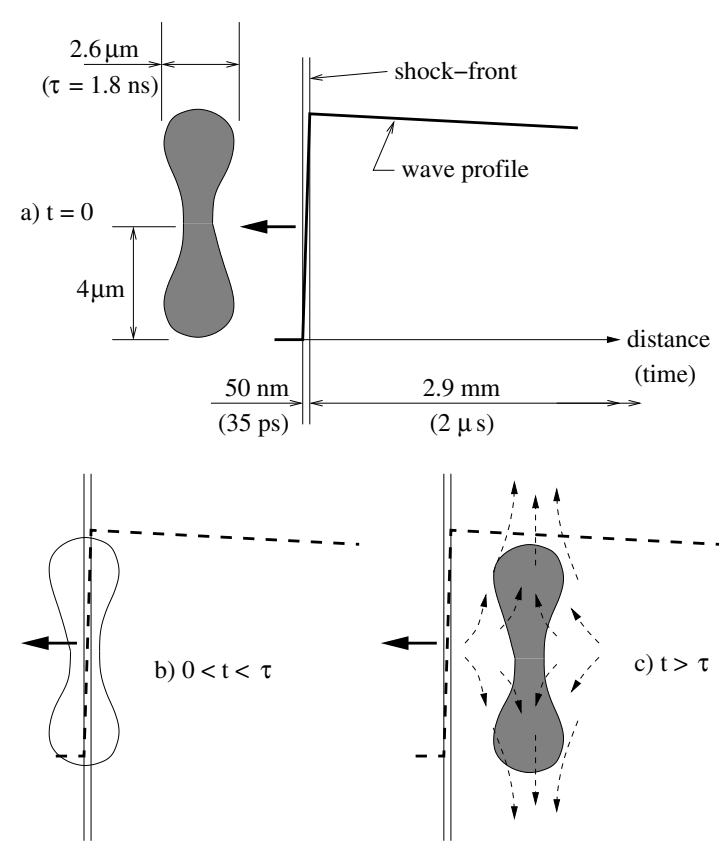

Figure 7. Illustrates the shock-RBC interaction, and the length(time) scales involved, drawn approximately to scale. Thick line indicates pressure (or velocity) profile of the wave. (a) $t=0$, before interaction, (b) $0<t<\tau$, (c) $t>\tau$, decaying flow-field. Dashed arrows represent the extensional flow-field induced by the shock.

\subsection{Shock-induced flow}

Figure 7 illustrates the interaction of the shock and the RBC. In figure 7(a) the length and timescales of this interaction are shown. For a shock strength of $40 \mathrm{MPa}$, the shock rise time, as obtained from Taylor's formula (Thompson 1972), is $\sim 35 \mathrm{ps,} \mathrm{This} \mathrm{translates} \mathrm{into} \mathrm{a} \mathrm{shock}$ thickness of $\sim 50 \mathrm{~nm}$. The steep wavefront eventually decays to the ambient conditions in around $2 \mu \mathrm{s}(\sim 2.9 \mathrm{~mm})$, and then becomes negative for $2-4 \mu \mathrm{s}$. In this work we will discuss the interaction with the positive wavefront only, and the extension to the negative portion can be done similarly. The RBC dimensions shown in figure 7 have been discussed earlier (section 2). The shock-RBC interaction can be differentiated into two time regimes (see figure 7):

- $0<t<\tau$. The time $\tau$ corresponds to the time required for the shock to propagate past a cell. Based on a cell width of $2.6 \mu \mathrm{m}, \tau=1.8 \mathrm{~ns}$. However, in general, the cell radius $\left(r_{c}=4 \mu \mathrm{m}\right)$ is a characteristic cell dimension, which gives $\tau \sim r_{c} / c \approx 3 \mathrm{~ns}$ $\left(c=1450 \mathrm{~m} \mathrm{~s}^{-1}\right)$. As the wavefront propagates through the cell, it induces a flow-field in and around the cell. The extensional flow is not fully developed, but comes into existence at the end of this time. Hence the analysis presented in this work is not strictly valid in this time regime, and the parameter $k(t)$, characterizing the extensional flow, has no meaning. However, we assume $k(t)$ to grow linearly during this regime to a maximum value, this maximum value being computed from measurements along the shock-front. Thus, the spatial gradient of the shock, along the direction of propagation, is approximated by a temporal variation in $k(t)$. It should be noted here that for situations where the shock rise time is greater than $3 \mathrm{~ns}$ the above assumption is not required and $\tau$ should be replaced by the shock rise time in the following analysis. 
- $t>\tau$. The extensional flow set up by the shock wave during the earlier time regime, decays in this time regime. Consequently, the tensions ( $T_{\text {iner }}$ and $\left.T_{\text {visc }}\right)$ also decay.

As explained in section 1.1, the lithotripter-generated focused shock wave has a curved wavefront of varying shock strength. Both the differential shock strength and the wavefront curvature result in an extensional flow. The parameter $k(t)$, due to both these effects, is now estimated. In a uniform fluid, the peak positive pressure, which is a measure of shock strength, drops from 40 to $0 \mathrm{MPa}$ within a distance of $1 \mathrm{~cm}$ (Cleveland et al 2000b). Thus the fluid particle velocity associated with this pressure $\left(u=\Delta p / \rho c=26 \mathrm{~m} \mathrm{~s}^{-1}\right)$ also has the same gradient. This gives us a maximum velocity gradient of $k_{\max }=26 / 0.01 \approx 3 \times 10^{3} \mathrm{~s}^{-1}$. This 'maximum' in $k$ is only a temporal maximum, spatially (over a beam width of $2 \mathrm{~cm}$ ) it is an averaged quantity. Since the shock-front is almost planar near the focus $k$ would have a spatial maximum away from the acoustic axis.

The curved shock-front induces a radial flow. Assuming that the shock-front is smooth (see section 7, discussion on 'kinks' in a shock-front), this radius will be of the order of the beam width, i.e. $2 \mathrm{~cm}$. Thus, the quantity $u_{R} / R$, characterizing the radial flow is of the same order as the quantity $k_{\max }$ calculated above for a shear flow. Hence in subsequent calculations, for shock-induced flows, we will disregard the radial flow, due to curvature of the focused shock, with the proviso that under conditions where shock curvature is significant its effect has to be accounted for. Thus $k(t)$ is defined as:

$$
\begin{aligned}
k(t) & =k_{\max } \frac{t}{3 \times 10^{-9}}, \quad 0 \leqslant t \leqslant 3 \times 10^{-9} \\
& =k_{\max }\left(1-\frac{t-3 \times 10^{-9}}{2 \times 10^{-6}}\right), \quad 3 \times 10^{-9} \leqslant t \leqslant 2 \times 10^{-6} \\
& =0, \quad t \geqslant 2 \times 10^{-6} .
\end{aligned}
$$

Figure $8(a)$ shows the velocity gradient $k(t)$ defined in equations (19)-(21). Inertial and viscous tensions are calculated using (7) and (15), and plotted in figures $8(b)$ and $8(c)$ respectively (note the change in scale of abscissa in figure 8 after $3 \times 10^{-9} \mathrm{~s}$ ). The maximum value of the tension due to inertial force is given by $\rho r_{c}^{3} k_{\max } / \tau \approx 64 \mathrm{mN} \mathrm{m}^{-1}$. During the decay period of the wave, this tension attains a value $0.1 \mathrm{mN} \mathrm{m}^{-1}$. Thus, the inertial force is strong enough to overcome the critical tension, but it lasts for a very brief time $\tau$. The areal strain induced during this time period, is $\Delta A / A \sim k_{\max } \tau \sim 10^{-5}$. Though the above strain is less than the critical areal strain of $10^{-2}$ (Evans et al 1976) needed for failure, it is sufficient for pores to form (see section 2). It should be noted here that the quantity $k_{\max } / \tau$, which determines the magnitude of inertial stress, is dependent on the pressure gradient in the direction of the shock propagation (since $\tau \sim$ shock rise time, for shock rise times $>3 \mathrm{~ns}$, and $k_{\max } \sim$ peak pressure/beam width). Hence a smaller shock rise time $(\tau)$ (i.e. steeper pressure front) would result in a higher $T_{\text {iner }}$.

The maximum viscous tension is given by $\rho(v / \pi \tau)^{1 / 2} r_{c}^{2} k_{\max } \approx 0.5 \mathrm{mN} \mathrm{m}^{-1}$, where $k_{\max } \approx 3 \times 10^{3} \mathrm{~s}^{-1}$ and $\tau \approx 3 \times 10^{-9} \mathrm{~s}$, as explained above. The tension decays from the maximum value in time $\sim \tau$. This tension is always less than the critical membrane tension, $T_{c}$. As discussed in section 1.1 , when $T_{\text {elas }} \ll T_{\text {iner }}$ the membrane deforms with the fluid, and there is negligible relative motion between the fluid and the membrane, i.e. $T_{\text {visc }} \approx 0$. Thus, the above calculation of $T_{\text {visc }}$ over $3 \mathrm{~ns}$ is an overestimate, and does not hold. However, after $T_{\text {iner }}$ has died out $(t>3 \mathrm{~ns})$, the viscous forces calculated here are valid. It should be noted that the maximum viscous tension calculated here is two orders higher than that obtained from a steady flow calculation $\left(T_{\mathrm{visc}, \mathrm{st}} \sim \mu k r_{c} \approx 10^{-2} \mathrm{mN} \mathrm{m}^{-1}\right)$. 


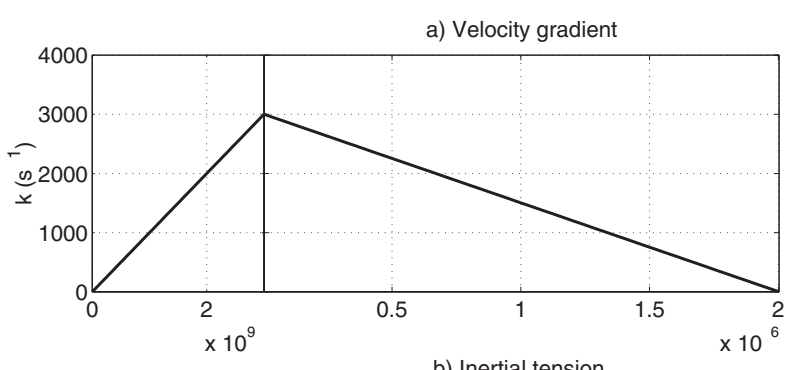

b) Inertial tension

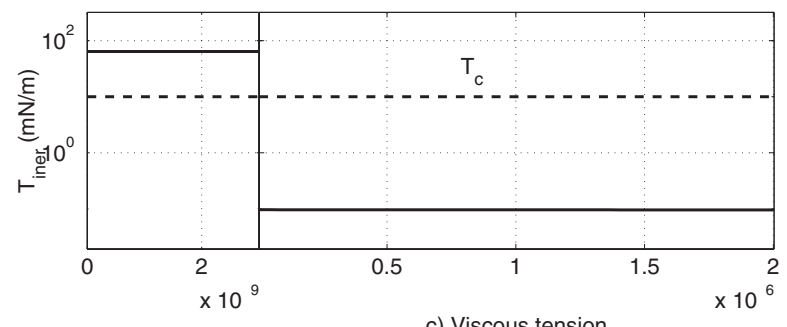

c) Viscous tension

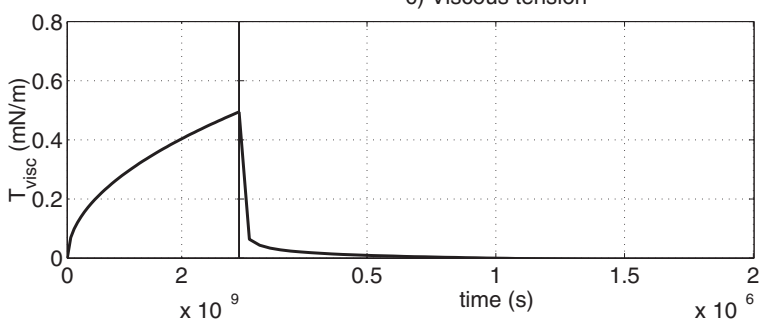

Figure 8. Membrane tension due to shock-induced shear flow. Note the change in scale of the abscissa after $3 \times 10^{-9} \mathrm{~s}$. (a) Velocity gradient as defined in (19). (b) Tension due to inertial forces, $T_{\text {iner }}$, as given by (7). The ordinate has a log scale. Dashed line indicates critical tension, $T_{c}$, for cell rupture. $(c)$ Tension due to viscous forces, $T_{\text {visc }}$, as given by (15).

\subsection{Bubble-induced flow}

The parameter $k(t)$ and the corresponding membrane tension for bubble-induced flow is calculated in this section. Rayleigh's model (Rayleigh 1917) is used for predicting the radial response of a single spherical bubble to a lithotripter pulse. The model problem is shown in figure 9. The equation of motion for bubble radius, $R_{b}$, is

$$
\ddot{R}_{b} R_{b}+\frac{3}{2} \dot{R}_{b}^{2}=\frac{1}{\rho}\left(p_{g}-p_{\infty}\right)
$$

where $p_{g}$ is the gas pressure inside the bubble, $\rho$ is the density of liquid surrounding the bubble and $p_{\infty}\left(=p_{0}+p_{\text {shock }}\right)$ is the far-field pressure, which comprises the ambient pressure $\left(p_{0}\right)$ and the shock pressure $\left(p_{\text {shock }}\right)$. The centre of the RBC is located at a radius $R_{\text {cell }}$ from the bubble centre. By definition (see section 3), $k(t)=U_{\text {cell }} / R_{\text {cell }}$, where $U_{\text {cell }}=\mathrm{d} R_{\text {cell }} / \mathrm{d} t$ is the radial velocity of the cell centre. The quantities $R_{\text {cell }}, U_{\text {cell }}$ are related to the bubble radius and bubble wall velocity $\left(U_{b}=\mathrm{d} R_{b} / \mathrm{d} t\right)$, by

$$
\begin{aligned}
& R_{\text {cell }}^{3}-R_{b}^{3}=R_{\text {cell0 }}^{3}-R_{b 0}^{3} \\
& U_{\text {cell }}=U_{b}\left(\frac{R_{b}}{R_{\text {cell }}}\right)^{2} .
\end{aligned}
$$




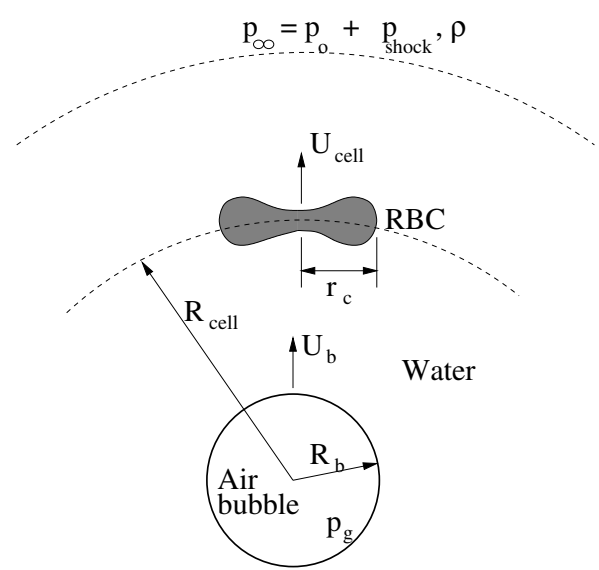

Figure 9. Model problem for bubble-RBC interaction.

Equation (23) is from volume conservation of an incompressible fluid. $R_{\text {cello }}$ is the initial radial location of the cell and $R_{b 0}$ is the initial bubble radius. Equation (24) is obtained by differentiating (23). The gas pressure inside the bubble is assumed to obey an isentropic law, $p_{g}=p_{0}\left(R_{b 0} / R_{b}\right)^{3 \gamma}$, where $\gamma=1.4$ is the ratio of the specific heats. The effects of surface tension and viscosity have been neglected. The Gilmore-Akulichev formulation has been used by others (Church 1989, Bailey et al 1999) for calculating the response of a bubble to lithotripter acoustic fields, but in this work the accuracy of the Rayleigh model is considered to be sufficient since only the preceding expansion phase of the bubble is being considered.

Figure 10 shows the results of the calculations. The differential equation (22) is integrated numerically using an explicit Runge-Kutta (4), (5) routine (known as ode45 in Matlab, MathWorks Inc., Natick, MA). A $5 \mu \mathrm{m}$ bubble is subjected to a tensile pulse of magnitude $5 \mathrm{MPa}$ and duration $5 \mu \mathrm{s}$. We ignore the positive pressure present in an actual SWL pulse, since it has a minimal effect on bubble growth which is due to the tensile portion only. As seen in figure $10(b)$, the bubble continually accelerates for the duration of application of the negative pulse $(5 \mu \mathrm{s})$, beyond which it starts to decelerate. Dashed lines in figure 10 indicate the radial location and velocity of a cell. The cell centre is initially located $40 \mu \mathrm{m}$ from the bubble centre $\left(R_{\text {cello }}\right)$. This initial location is selected to satisfy the assumption $r_{c} / R_{\text {cello }} \ll 1$, which was made while linearizing the radial flow to an extensional flow (see the appendix). Membranes of cells closer to the bubble will be subjected to stronger forces than that calculated here, but these cannot be estimated accurately due to the approximations made here. As seen in figure $10(a)$, the difference between the cell radial location $\left(R_{\text {cell }}\right)$ and the bubble radius $\left(R_{b}\right)$ can be seen for a very short time only $(2-3 \mu \mathrm{s})$. Similarly the radial cell velocity, $U_{\text {cell }}$, differs from the bubble wall velocity for the first few $\mu$ s only.

The parameter of interest, $k(t)$, which characterizes the extensional flow in the frame of reference of the cell, is plotted in figure $11(a)$. It reaches a maximum $\left(k_{\max }\right)$ of $7 \times 10^{5} \mathrm{~s}^{-1}$ at $\tau \approx 0.9 \mu \mathrm{s}$. This gives an inertial tension of $100 \mathrm{mN} \mathrm{m}^{-1}$ (seen from figure $11(b)$ ). Using the estimates from table 1, the unsteady and steady inertial tensions can be calculated as $64 \mathrm{mN} \mathrm{m}^{-1}$ and $31 \mathrm{mN} \mathrm{m}^{-1}$, respectively. Thus the bubble-induced flow exerts an inertial force of the same order as the shock-induced flow, both of them higher than the membrane critical tension (figure $8(b)$ ). However, in this case this force lasts for a duration of $1 \mu \mathrm{s}$, causing an areal strain of $\Delta A / A=k_{\max } \tau \approx 1$ (or $100 \%$ ), two orders higher than the required strain of 2-3\% for failure. 

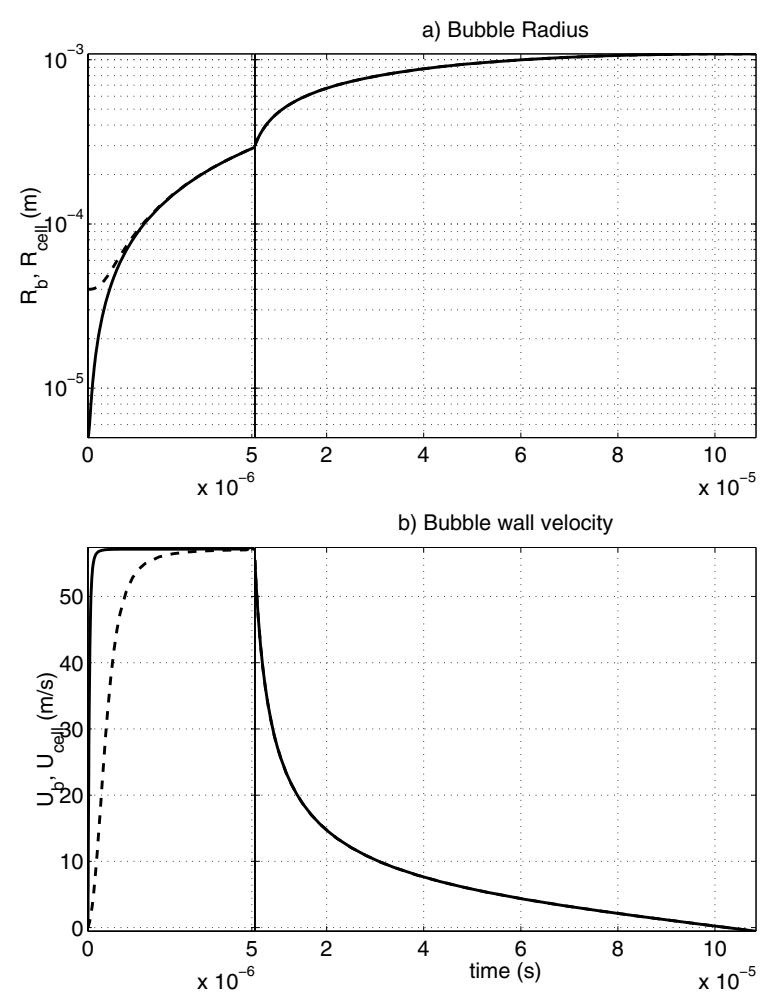

Figure 10. Response of a $5 \mu \mathrm{m}$ bubble from Rayleigh's model. Bubble subjected to a tensile pulse of $5 \mathrm{MPa}$ for a duration of $5 \mu \mathrm{s}$. Note the change in scale of the abscissa after $5 \times 10^{-6} \mathrm{~s}$. (a) Bubble radius $\left(R_{b}\right)$ (full curve). Dashed curve corresponds to radial location of a cell $\left(R_{\text {cell }}\right)$, initially at $40 \mu \mathrm{m}$ from the bubble centre. $(b)$ Bubble wall velocity $\left(U_{b}\right)$. Dashed curve corresponds to radial cell velocity $\left(U_{\text {cell }}\right)$.

Viscous tension in the absence of inertial deformation is plotted in figure 11(c), for the sake of completeness. As discussed for the shock-induced flow, the cell in this case will deform in accordance with the inertial forces, and there will be negligible relative motion between the cell and the extensional flow. Consequently $T_{\text {visc }} \approx 0$ for the first $1 \mu \mathrm{s}$, contrary to the plotted values.

\section{Discussion}

Experiments show that in vitro exposure of RBCs to SWL shock waves, when suspended in either a cavitating or a non-cavitating environment, cause the cells to lyse. In this work we examined the relation between an arbitrary flow-field and the deformation of a cell immersed in it, and applied this analysis to SWL flow-fields. The fluid motion is kinematically decomposed into extensional, translational and rotational motions. Since only the extensional motion causes the cell to deform, that component must be large enough if the flow is to be lytically effective. In SWL, a focused shock wave, as well as radial flow due to an expanding/imploding bubble, both comprise an unsteady extensional flow, causing cells to deform.

The inertial and viscous forces exerted on the cell membrane by an unsteady extensional flow are estimated. The deformation parameter $(k)$ and the time scale $(\tau)$ of the flow-field are 


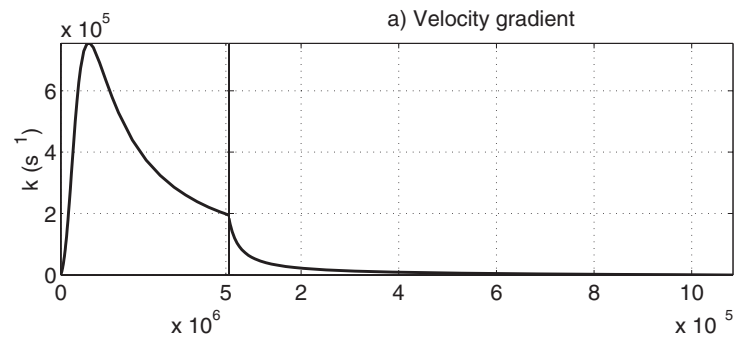

b) Inertial tension

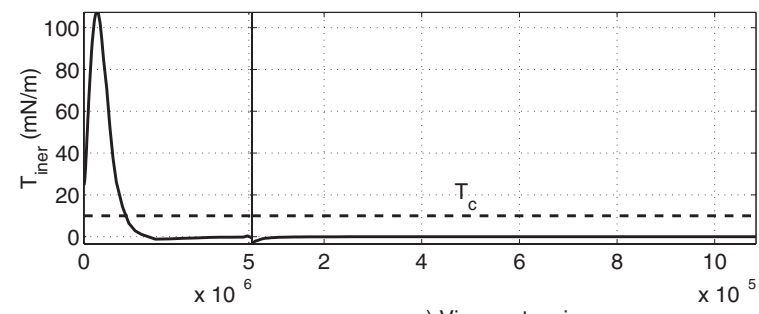

c) Viscous tension

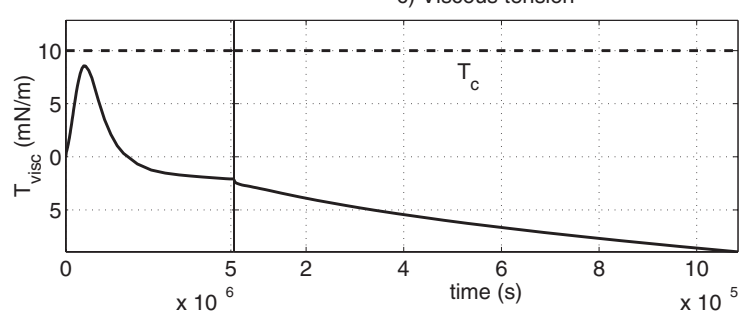

Figure 11. Membrane tension due to bubble-induced radial flow. Note the change in scale of abscissa after $5 \times 10^{-6} \mathrm{~s}$. (a) Velocity gradient as defined in (19). (b) Tension due to inertial forces, $T_{\text {iner }}$, as given by (7). Dashed line indicates critical tension, $T_{c}$, for cell rupture. $(c)$ Tension due to viscous forces, $T_{\mathrm{visc}}$, as given by (12).

the important parameters, which govern the magnitudes of the inertial and viscous forces on the cell membrane. Figure $6(b)$ is reinterpreted in figure 12, and summarizes our analytical results. As explained earlier, the membrane deformation can be either subcritical, i.e. a transient population of pores can arise in the membrane, or critical, i.e. the membrane can rupture irreversibly, resulting in cell lysis. The solid portions of the lines $\Delta A / A=2.5 \%$ and $T_{\text {visc }}=T_{c}$ demarcate the regimes of subcritical and critical cell deformation, on the $k-\tau$ plane (figure 12), marked as 'poration' and 'lysis' respectively. In the 'poration' regime cell deformation is not sufficient for lysis but will enhance transport across the cell membrane. This would clearly reduce as we move farther away from the above lines (in the direction of reducing $k$ ). Since the parameters $k$ and $\tau$ determine the lytic capabilities of a flow-field, in figure 12 representative $k-\tau$ values for a range of experimental setups have been superposed on our analytically determined cell deformation/lysis regimes. These experimental results are shown in table 2, and are discussed briefly.

As discussed in section 2, viscometers have been more commonly used for measuring cell lysis. Typical values of $k$ for viscometers (e.g. Leverett et al 1972) are $10^{4}-10^{5} \mathrm{~s}^{-1}$. These are represented by the shaded region marked 1 in figure 12. The parameter $\tau$ (time taken by the viscometer to accelerate to the maximum speed) is usually $\sim 1-10 \mathrm{~s}$, and hence the steady shearing effect dominates in this regime of operation. Leverett et al (1972) have summarized a plot of threshold shear stress for lysis versus duration of exposure, which would 


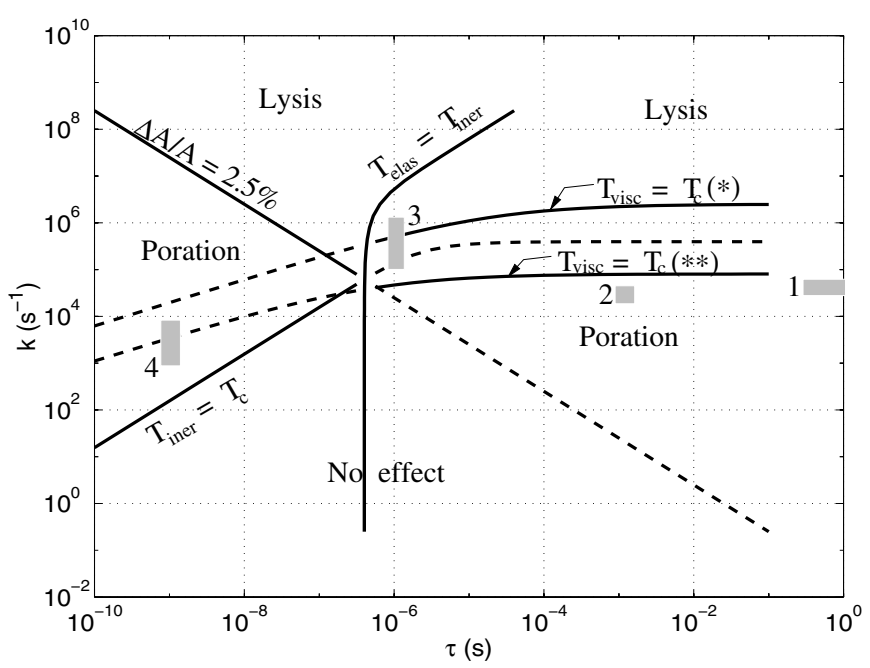

Figure 12. Cell deformation/lysis regimes on a $k-\tau$ plot. Shaded areas (marked by numbers) represent operating regimes of various experimental setups, tabulated in table 2 (see corresponding number in table). * Water is the suspending fluid, $\mu=10^{-3} \mathrm{~Pa} \mathrm{~s}$; ** suspending fluid has $\mu=31 \times 10^{-3}$ Pa s (Rooney 1972).

Table 2. Summary table of various experiments on RBC lysis, listing $k-\tau$ parameter of their flow-fields, and deformation/lysis mode(s).

\begin{tabular}{llllll}
\hline & & & & $\begin{array}{l}\text { Deformation/ } \\
\text { lysis modes }\end{array}$ & References \\
\hline 1 & Viscometer & $10^{4}-10^{5}$ & $1-10$ & Viscous & Leverett 1972 \\
2 & Single bubble & $10^{4}-10^{5}$ & $10^{-3}$ & Inertial/Viscous & Rooney 1972 \\
3 & $\begin{array}{l}\text { Cavitation } \\
\dagger\end{array}$ & $10^{6}$ & $10^{-6}$ & Inertial/Viscous & $\begin{array}{l}\text { Delius 1997 } \\
\text { (SWL) }\end{array}$ \\
& & & & Stonehill 1998 \\
4 & $\begin{array}{l}\text { Shock wave } \\
\text { (SWL) }\end{array}$ & $10^{3}-10^{4}$ & $10^{-9}$ & Inertial & Williams 1999 \\
& & & & Lokhandwalla 2001 \\
\hline
\end{tabular}

$\dagger_{k-\tau}$ values are estimated in this work.

be identical to figure 12 , for $\tau \gg 10^{-6} \mathrm{~s}$ (since $k$ multiplied by viscosity yields shear stress, and $\tau$ would mean duration of exposure). Other analytical/computational work (e.g. Ramanujan and Pozrikidis 1998) has considered cells subjected to steady (or quasisteady) shear flow, at very low Reynolds number (Stokes' flow), which is applicable to typical physiological flow conditions, but not to SWL flow-fields dealt with here $(R e>1)$. Acoustic transients could also induce a Stokesian flow-field (e.g. Hamrick and Cleary 1969) when the acoustic velocity and the cell size under consideration are sufficiently small, resulting in a low Reynolds number, contrary to the conditions considered here. In figure 12 the shaded area marked 2 represents cell lysis experiments performed by Rooney $(1970,1972)$. In Rooney's experiments a single bubble was set into translational motion by an acoustic field in a suspending fluid of viscosity $0.031 \mathrm{~Pa}$ s. A steady streaming motion is set up due to the translational motion of the bubble, which was explained to cause cell lysis.

The inertial tension due to a bubble-induced radial flow-field during its expansion phase was calculated to be $100 \mathrm{mN} \mathrm{m}^{-1}$ (section 6). This force is much higher than the threshold membrane tensions measured in single cell experiments. Also, the calculations suggest a strain 
of $\sim 100 \%$, which means that the surface area will be stretched to twice the original area, well beyond the critical strains measured in experiments. In figure 12 the calculated values of $k$ and $\tau$, for bubble-induced radial motion, are marked 3. It should be noted that although 3 lies in the viscous deformation regime, it is located proximally to the transition line $\left(T_{\text {elas }}=T_{\text {iner }}\right)$, and hence cell deformation/lysis is due to inertial forces. In the above calculation we assumed the cell to lie at a particular radial distance from the bubble centre $\left(R_{\text {cello }}=40 \mu \mathrm{m}\right)$. Cells farther away from the bubble will be subjected to smaller magnitudes of forces and strains, and will consequently have a lower probability of failure. Hence in the region surrounding the bubble, cell lysis is readily explained in terms of the radial motion of a bubble set up by the tensile phase of the lithotripter pulse.

The inertial tension due to the shock-induced flow-field was calculated to be $64 \mathrm{mN} \mathrm{m}^{-1}$. Though this force is sufficient to deform the membrane, it lasts for a very short time, $\sim 3 \mathrm{~ns}$. The strain induced by the inertial forces is $\sim 10^{-5}$, much lower than the critical strain, $\sim 10^{-2}$, needed to rupture the cells but larger than that required to induce pores, $\sim 10^{-7}-10^{-5}$, as described in section 2. Thus, the strain is sufficient to induce pores, but since it is much lower than the critical strain, the probability that these pores grow unstably and cause the membrane to rupture is very low, i.e. only a fraction of all the cells subjected to shock waves will be lysed. To estimate this fraction would require the analysis of membrane dynamics beyond the pore formation stage, which is beyond the scope of this work. The area marked 4 in figure 12 represents shock-induced flow parameters. Also while calculating the shockinduced deformation, the effect of wavefront curvature was ignored. For focused waves with a finite amplitude this is an important effect (Sturtevant 1996). A converging wavefront of finite amplitude is unstable, and forms sharp corners or 'kinks' equivalent to Mach reflection. The inherent variability of the lithotripter acoustic field (Cleveland et al 2000b), makes it difficult to confirm the absence or presence of these sharp corners in the shockfront. However, the presence of these 'kinks' in the shockfront would suggest that the deformation parameter $(k(t))$ is several orders higher, leading to higher lytic effects in these small regions.

An important assumption in our analysis is that the effect of the shock gradient in the direction of propagation can be approximated by a temporal variation in the flow-field $(\dot{k})$ past the cell. Thus, a steeper shock gradient (higher $\dot{k}$ ) results in a higher value of $T_{\text {iner }}$. The effect of the shock gradient on cell lysis has been studied experimentally by Doukas et al (1995), using laser-generated stress waves. These investigators found a higher cell lysis with an increasing stress gradient in the direction of wave propagation. The stress wave propagating through their cell sample was assumed to be uniform and planar. Their result would corroborate our hypothesis provided their stress wave is found to have a curvature or to be of non-uniform strength.

We conclude this work by proposing a general perspective on the mechanisms of cell lysis, as shown in figure 13. As discussed in section 2, the bilayer configuration is a metastable state of the membrane, and membrane rupture can be effected by providing a sufficient amount of energy. Hence a very general classification of cell lysis mechanisms is based on the means of providing this energy, i.e. thermal (or radiation) or mechanical. In mechanical modes, the cell membrane is subjected to a tension by mechanical means, for example the cell aspirator (figure 3) discussed earlier. A cell could be entirely submerged in a fluid medium, without any contact with a solid surface, or could be tethered to (or in contact with) a solid surface. For cells submerged in a fluid medium, the membrane could be subjected to static forces (for example osmotic pressure) which do not arise due to bulk fluid motion. When there is bulk fluid motion, fluid dynamic forces are exerted on the cell membrane. These fluid dynamic forces are primarily inertial and viscous forces, either due to a steady or an unsteady flow. Cell lysis due to microstreaming or cell lysis in viscometers is classified into the viscous 


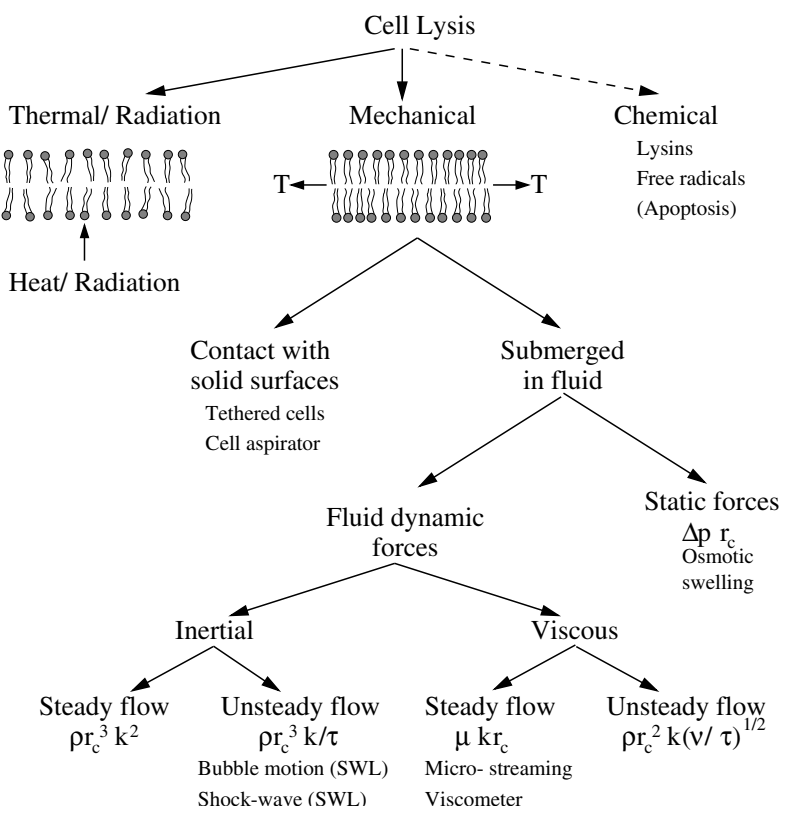

Figure 13. A general perspective of cell lysis mechanisms.

steady-flow category, whereas the bubble-induced and shock-induced SWL flow-fields belong to the inertial, unsteady category. Though the primary motivation of this work was to analyse cell lysis due to SWL induced flow-fields, we have also demonstrated a means of systematically classifying all the previously reported modes of mechanical lysis based on the nature of the predominant force. A better understanding of the interaction between the flow-field and the cell membrane and membrane poration will enable us to analyse/control membrane poration and concomitant exchange of extra- and intracellular material. A variety of related problems, including localized drug delivery using shock waves, increased haemolysis in prosthetic heart valves (Ellis et al 1998) have a potential gain from such an improved understanding.

\section{Acknowledgments}

We thank Ganesh Subramanian of California Institute of Technology and Dr Jim Williams of Indiana University School of Medicine for their valuable comments on the ideas discussed in this work. This work was supported by NIH Grant P01 DK43881.

\section{Appendix. Decomposition of radial flow-field}

Three-dimensional radial flow is given by

$$
\vec{u}_{r}=\frac{Q(t)}{4 \pi r^{2}} \vec{e}_{r}
$$

where $Q(t)$ represents an unsteady source term at $r=0, r$ is the radial coordinate with respect to the source location, (in figure $\mathrm{A} 1(a)$, the source is located at the origin of the $(x, y, z)$ reference system) and $\vec{e}_{r}$ is a unit vector along the radial direction (oa). The coordinate transformation from the fixed frame of reference, $(x, y, z)$, to the moving frame of reference 


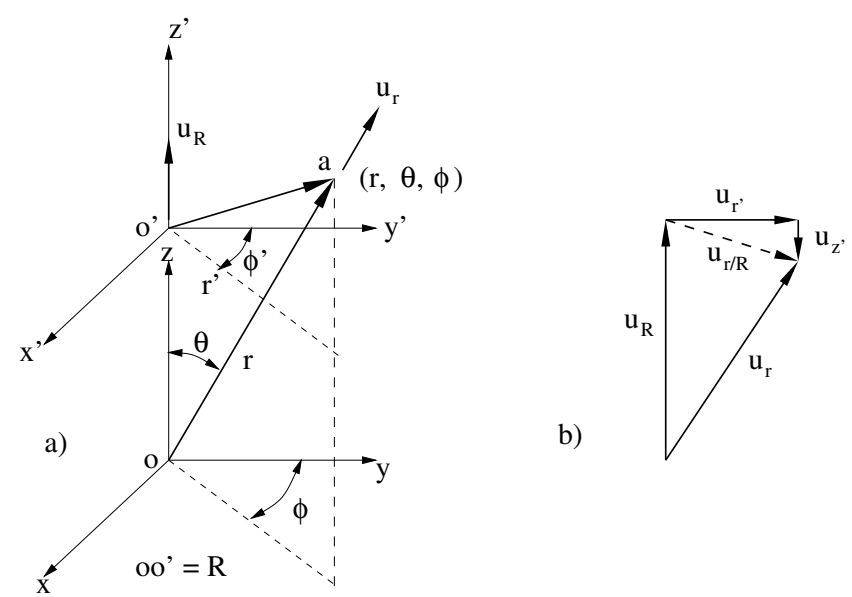

Figure A1. Radial flow decomposition. (a) The coordinate system $(x, y, z)$ is fixed in space, with the source at its origin; $\left(x^{\prime}, y^{\prime}, z^{\prime}\right) \equiv\left(r^{\prime}, z^{\prime}, \phi^{\prime}\right)$ is moving with the particle velocity $u_{R}$. (b) Resolving velocity components in the moving coordinate system $\left(r^{\prime}, z^{\prime}, \phi^{\prime}\right)$.

$\left(x^{\prime}, y^{\prime}, z^{\prime}\right)$ (this is equivalent to $\left.\left(r^{\prime}, z^{\prime}, \phi^{\prime}\right)\right)$ is given by

$$
\left(\phi^{\prime}, r^{\prime}, z^{\prime}\right)=(\phi, r \sin \theta, r \cos \theta-R) .
$$

Non-dimensionalizing $r^{\prime}, z^{\prime}$ by $R$ and denoting them as $r^{\prime \prime}, z^{\prime \prime}$, we get

$$
\begin{aligned}
& r^{2}=R^{2}\left(\left(z^{\prime \prime}+1\right)^{2}+r^{\prime \prime 2}\right) \\
& \cos \theta=\left(z^{\prime \prime}+1\right) /\left(\left(z^{\prime \prime}+1\right)^{2}+r^{\prime \prime 2}\right)^{1 / 2} \\
& \sin \theta=r^{\prime \prime} /\left(\left(z^{\prime \prime}+1\right)^{2}+r^{\prime \prime 2}\right)^{1 / 2} .
\end{aligned}
$$

For brevity, the double primes (") over normalized variables will be dropped. Using the above transformation, (A.1) can be written as

$$
\vec{u}_{r}=\frac{Q(t)}{4 \pi R^{2}} \frac{\left(\vec{e}_{r^{\prime}} \sin \theta+\vec{e}_{z^{\prime}} \cos \theta\right)}{(z+1)^{2}+r^{2}}
$$

where $\vec{e}_{r^{\prime}}, \vec{e}_{z^{\prime}}$ are unit vectors along $r^{\prime}, z^{\prime}$ directions shown in figure A1(a). The velocity of the moving reference frame $\left(x^{\prime}, y^{\prime}, z^{\prime}\right)$ is $\vec{u}_{R}(t)=Q(t) /\left(4 \pi R^{2}\right) \vec{e}_{z^{\prime}}$. The components of the velocity field, given by (A.6), expressed in the moving reference frame, is given by

$$
\begin{aligned}
& u_{r_{R}}=u_{R}(t)\left(\frac{r}{\left((z+1)^{2}+r^{2}\right)^{3 / 2}}\right) \\
& u_{z_{R}}=u_{R}(t)\left(\frac{z+1}{\left((z+1)^{2}+r^{2}\right)^{3 / 2}}-1\right)
\end{aligned}
$$

where the subscript $r_{R}, z_{R}$ indicates the $r, z$ components relative to the moving frame. Linearizing the velocity components, i.e. assuming $r, z \ll 1$, (A.7) and (A.8) can be written as, consistent with $\mathcal{O}\left(r^{2}, z^{2}, z r\right)$

$$
\begin{aligned}
& u_{r_{R}}=u_{R}(t)(r-3 z r) \\
& u_{z_{R}}=u_{R}(t)\left(-2 z+3 z^{2}-\frac{3}{2} r^{2}\right) .
\end{aligned}
$$

The above equations can be checked to satisfy the continuity equation

$$
\frac{1}{r} \frac{\partial\left(r u_{r_{R}}\right)}{\partial r}+\frac{\partial\left(u_{z_{R}}\right)}{\partial z}=0
$$


and the condition for potential flow, $\vec{u}=\nabla \phi$, with the velocity potential being

$$
\phi(t)=R u_{R}(t)\left(\frac{r^{2}}{2}-z^{2}-\frac{3}{2} z r^{2}+z^{3}\right) .
$$

For a shear flow

$$
\vec{u}(x, y, z)=U(1,0,0)+(k(t) y, 0,0)
$$

the decomposition is fairly trivial. Equation (A.13) can be written as

$$
\vec{u}(x, y, z)=U(1,0,0)+\left(\frac{k(t)}{2} y, \frac{-k(t)}{2} x, 0\right)+\left(\frac{k(t)}{2} y, \frac{k(t)}{2} x, 0\right) .
$$

The three terms on the right-hand side of (A.14) represent the following respectively: uniform translation, rotation about the $z$-axis and an extensional flow, with the principal axes of deformation oriented at $45^{\circ}$ to the $(x, y)$ coordinate system. The linearized $(\mathcal{O}(r, z)$ terms only) version of (A.9) and (A.10), is an axisymmetric extensional flow in the $(r, z)$ plane, whereas the last bracketed term in (A.14) represents planar extensional flow (in the $(x, y)$ plane).

\section{References}

Aris R 1989 Vectors, Tensors, and the Basic Equations of Fluid Mechanics (New York: Dover)

Bailey M R, Blackstock D T, Cleveland R O and Crum L A 1999 Comparison of electrohydraulic lithotripters with rigid and pressure-release ellipsoidal reflectors: II. Cavitation fields J. Acoust. Soc. Am. 106 1149-60

Barthes-Biesel D 1980 Motion of a spherical microcapsule freely suspended in a linear shear flow J. Fluid Mech. 100 831-53

Blake J R and Gibson D C 1987 Cavitation bubbles near boundaries Ann. Rev. Fluid Mech. 1999-123

Carstensen E L, Kelly P, Church C C, Brayman A A, Child S Z, Raeman C H and Schery L 1993 Lysis of erythrocytes by exposure to CW ultrasound Ultrasound. Med. Biol. 19 147-65

Church C C 1989 A theoretical study of cavitation generated by an extracorporeal shockwave lithotripter J. Acous. Soc. Am. 86 215-27

Cleveland R O, Bailey M R, Fineberg N, Hartenbaum B, Lokhandwalla M, McAteer J A and Sturtevant B 2000b Design and characterization of a research electrohydraulic lithotripter patterned after the Dornier HM3 Rev. Sci. Instrum. 71 2514-25

Cleveland R O, Sapozhnikov O A, Bailey R M and Crum L A 2000a A dual passive cavitation detector for localized detection of lithotripsy-induced cavitation in vitro J. Acoust. Soc. Am. 107 1745-58

Coleman A J and Saunders J E 1993 A review of the physical properties and biological effects of the high amplitude acoustic fields used in extracorporeal lithotripsy Ultrasonics 31 75-89

Delius M 1997 Minimal static pressure minimizes the effect of extracorporeal shock waves on cells and reduces it on gallstones Ultrasound Med. Biol. 23 611-17

Doukas A G, McAuliffe D J, Lee S, Venugopalan V and Flotte T 1995 Physical factors involved in stress-wave-induced cell injury: the effect of stress gradient Ultrasound Med. Biol. 21 961-7

Ellis J T, Wick T M and Yoganathan A P 1998 Prosthesis-induced hemolysis: mechanisms and quantification of shear stress J. Heart Valve Dis. 7 376-86

Evan A P and McAteer J A 1996 Q-Effects of shock wave lithotripsy Kidney Stones: Medical and Surgical Management ed F L Coe et al (Philadelphia: Lippincott-Raven) pp 549-70

Evans E A and Fung Y C 1972 Improved measurements of the erythrocyte geometry Microvasc. Res. 4 335-47

Evans E A, Waugh R and Melnik L 1976 Elastic area compressibility modulus of red cell membrane Biophys. J. 16 585-95

Hamrick P E and Cleary S F 1969 Breakage of tobacco mosaic virus by acoustic transients: a hydrodynamical model J. Acoust. Soc. Am. 45 1-6

Howard D D and Sturtevant B 1997 In vitro study of the mechanical effects of shock wave lithotripsy Ultrasound Med. Biol. 23 1107-22

Kaude J V, Williams C M, Millnder M R, Scott K N and Finlayson B 1985 Renal morphology and function immediately after extracorporeal shock-wave lithotripsy Am. J. Roentgenol. 145 305-13

Leverett L B, Hellums J D, Alfrey C P and Lynch E C 1972 Red blood cell damage by shear stress Biophys. J. 12 $257-73$ 
Li X Z, Barthes-Biesel D and Helmy A 1988 Large deformations and burst of a capsule freely suspended in an elongational flow J. Fluid. Mech. 187 179-96

Litster J D 1975 Stability of lipid bilayers and red blood cell membranes Phys. Lett. A 53 193-4

Lokhandwalla M 2001 Damage mechanisms in shock wave lithotripsy PhD Thesis California Institute of Technology, Pasadena, CA

Lokhandwalla M, McAteer J A, Williams J C Jr and Sturtevant B 2001 Mechanical haemolysis in shock wave lithotripsy (SWL): II. Cell lysis due to shear Phys. Med. Biol. submitted

Miller M W, Miller D L and Brayman A A 1996 A review of in vitro bioeffects of inertial ultrasonic cavitation from a mechanistic perspective Ultrasound Med. Biol. 22 1131-54

Mulholland S E, Lee S, McAuliffe D J and Doukas A G 1999 Cell loading with laser-generated stess waves: the role of the stress gradient Pharm. Res. 16 514-18

Needham D and Hochmuth R M 1989 Electro-mechanical permeabilization of lipid vesicles-role of membrane tension and compressibility Biophys. J. 55 1001-9

Neumann E and Rosenheck K 1972 Permeability changes induced by electric impulses in vesicular membranes J. Membrane Biol. 10 279-90

Pozrikidis C 1997 Introduction to Theoretical and Computational Fluid Dynamics (New York: Oxford University Press)

Ramanujan S and Pozrikidis C 1998 Deformation of liquid capsules enclosed by elastic membranes in simple shear flow: large deformations and the effect of fluid viscosities J. Fluid. Mech. 361 117-43

Rand R P 1964 Mechanical properties of the red cell membrane: II. Viscoelastic breakdown of the membrane Biophys. J. 4 303-16

Rand R P and Burton A C 1964 Mechanical properties of the red cell membrane: I. Membrane stiffness and intracellular pressure Biophys. J. 4 115-35

Rayleigh J W 1917 On the pressure developed in a liquid during the collapse of a spherical cavity Phil. Mag. 34 94-8

Rooney J A 1970 Hemolysis near an ultrasonically pulsating gas bubble Science 169 869-71

-1972 Shear as a mechanism for sonically induced biological effects J. Acoust. Soc. Am. 52 1718-24

Shillcock J C and Boal D H 1996 Entropy driven instability and rupture of fluid membranes Biophys. J. 71 317-26

Stonehill M A, Williams J C, Bailey M R, Lounsbery D, Cleveland R O, Crum L A, Evan A P and McAteer J A 1998 An acoustically matched high pressure chamber for control of cavitation in shockwave lithotripsy: Mechanism of shock wave damage in vitro Methods Cell Sci. 19 303-11

Sturtevant B 1996 Shock wave physics of lithotripters Smith's Textbook of Endourology ed A D Smith et al (St Louis, MO: Quality Medical) pp 529-52

Teshima K, Ohshima T, Tanaka S and Nagai T 1995 Biomechanical effects of shock waves on Escherichia coli and $\lambda$ phage DNA Shock Waves 4 293-7

Thompson P A 1972 Compressible Fluid Dynamics (New York: McGraw Hill)

Truesdell C A 1954 The Kinematics of Vorticity (Bloomington, IN: Indiana University Press)

Weaver J C 1995 Electroporation theory: concepts and mechanisms Methods in Molecular Biology, Vol 47: Electroporation Protocols for Microorganisms ed J A Nickoloff (Totowa NJ: Humana Press) pp 1-26

Williams J C, Woodward J F, Stonehill M A, Evan A P and McAteer J A 1999 Cell damage by lithotripter shock waves at high pressure to preclude cavitation Ultrasound Med. Biol. 25 1445-9 